DOI: https://doi.org/10.46296/yc.v5i9edespdic.0141

\title{
REGRESIÓN DE COX PARA SUPERVIVENCIA EN PACIENTES CON CÁNCER COLORRECTAL
}

\section{COX REGRESSION FOR SURVIVAL IN COLORECTAL CANCER PATIENTS}

\author{
Molina Miguel Ángel ${ }^{\text {; }}$ Alarcón-Cano Daniel Fabricio ${ }^{2}$; \\ Montes-Escobar Karime ${ }^{3}$ \\ ${ }^{1}$ Maestría en Estadística, Instituto de Posgrado, Universidad técnica de Manabí, UTM. \\ Portoviejo, Ecuador. Correo: mmolina3227@utm.edu.ec. \\ ${ }^{2}$ Departamento de Docencia e investigación, SOLCA. Portoviejo, Ecuador. Correo: \\ alarcon.daniel.md@gmail.com. \\ ${ }^{3}$ Departamento de Matemáticas y Estadística. Instituto de Ciencias Básicas, Universidad \\ Técnica de Manabí, UTM. Portoviejo, Ecuador. Correo: karime.montes@utm.edu.ec.
}

\begin{abstract}
Resumen
Objetivo: Determinar la supervivencia en pacientes con cáncer colorrectal mediante el modelo de Regresión de Cox a 6 años, periodos 2010 - 2015 en la Provincia de Manabí, Ecuador. Estudio observacional, descriptivo y retrospectivo, se analizaron los datos de 129 pacientes diagnosticados con cáncer colorrectal para el análisis de supervivencia. Las variables en la investigación fueron: Edad, sexo, estadio de la enfermedad (Tx, T2, T3 y T4) y supervivencia. Se instruyen fundamentos del modelo de Cox basada en la denominada "razón de riesgos" o estadística Hazard Ratio HR. De los 129 pacientes, la edad media fue de 61,6 años, con un rango de 14 a 97 años, el sexo femenino obtuvo un mayor porcentaje con 55,81\% pacientes y el masculino con 44,19\% pacientes. Se examinó los estadios donde la mayor frecuencia es TNM 3 con $49,61 \%$, seguido del TNM x con $27,13 \%$, la supervivencia global fue de $15 \%$ a 72 meses. EI estadio de la enfermedad (tumor) es la que estadísticamente estuvo asociada significativamente a la supervivencia, donde no evaluar el tumor principal (TNMx) llamó la atención en este estudio. No efectuar diagnósticos a tiempos y llegar a estadios tardíos disminuye la supervivencia en el cáncer colorrectal en la Provincia de Manabí.
\end{abstract}

Palabras claves: modelo de riesgos proporcionales de Cox, supervivencia, cáncer de estómago, Ecuador.

\begin{abstract}
To determine survival in patients with colorectal cancer using the Cox Regression model at 6 years, periods 2010 -2015 in the Province of Manabí, Ecuador. Observational, descriptive, and retrospective study, the data of 194 patients diagnosed with colorectal cancer were analyzed. Under inclusion criteria, 129 patients remained for survival analysis. The variables included in the research were: age, sex, stage of the disease (Tx, T2, T3 and T4) and survival. The basics of the Cox Regression model are instructed based on the so-called "risk ratio" or Hazard Ratio HR statistic. Of the 129 patients, the mean age was 61.6 years, with an age range of 14 to 97 years, with a superiority of the female sex with $55.81 \%$ patients and the male with $44.19 \%$. The stages where the highest frequency is TNM 3 with $49.61 \%$, followed by TNM $\times$ with $27.13 \%$, were examined, the overall survival was $15 \%$ at 72 months. The stage of the disease (tumor) is the one that was statistically significantly associated with survival, where not evaluating the main tumor attracted attention in this study, that is, TNM stage $x$. It can be concluded that not making diagnoses in time and reaching late stages decreases survival in colorectal cancer in the Province of Manabí.
\end{abstract}

Keywords: Cox proportional hazards model, survival, stomach cancer, Ecuador.

Información del manuscrito:

Fecha de recepción: 29 de octubre de 2021.

Fecha de aceptación: 19 de noviembre de 2021.

Fecha de publicación: 17 de diciembre de 2021. 


\section{Introducción}

Este estudio da lugar a una línea de investigación donde se desea determinar el impacto que generan las variables estadio de la enfermedad, edad, sexo, de manera individual en la personas o pacientes que padecen de cáncer colorrectal enlazado con la supervivencia a seis años en la Provincia de Manabí. En base a datos de Globocan en el Ecuador el cáncer colorrectal en los hombres es la tercera neoplasia y en las mujeres la quinta lo que significa un problema de salud pública (Ministerio de Salud Pública, 2017); en el año 2013 el cáncer colorrectal en la ciudad de Quito tuvo un aumento significativo tanto en hombres (CPA de 2.5), como en mujeres, (CPA 1.4); cifras muy semejantes para ambos sexos. La mortalidad, en hombres y mujeres estuvieron al alza significativamente (3.5 y 4.2, respectivamente) (Corral et al., 2018).

Actualmente el cáncer colorrectal figura como la tercera razón de incidencia y la segunda en mortalidad en hombres y mujeres a nivel mundial, con una mayor frecuencia en los hombres
(Villanueva-Pájaro et al., 2020). En países desarrollados la probabilidad de percibir cáncer de colorrectal en algún momento de la vida es de 4.6 $\%$ para hombres y $3.2 \%$ para mujeres, siendo los de mayor incidencia países tales como: Australia, Nueva Zelanda, Norteamérica y Europa Occidental. Estos territorios se han denominado de alto riesgo, y en varios de ellos la incidencia y mortalidad por cáncer de colon, tienden hacia la estabilización o disminución; fenómeno atribuible al desarrollo de estrategias de tamizaje y diagnóstico de lesiones precancerosas, asociado a un tratamiento agresivo y oportuno (Oviedo, 2015).

Asimismo, de acuerdo con Globocan 2012, el valor de incidencia del CCR en hombres es de 10 y en mujeres de 11.3 por 100.000 habitantes, viéndose una tendencia en ascenso. Conforme al Registro Nacional de Tumores 2014 de SOLCA, en el año 2012 murieron 603 personas en Ecuador por cáncer colorrectal, la incidencia es creciente en hombres y en mujeres. Conforme con la medicina basada en la evidencia, la sobrevida de los pacientes a 5 años es del $90 \%$ con CCR temprano y con 
CCR avanzado es del 8 al 15\%, he aquí la importancia de encontrar los daños cuando todavía son curables (Castillo Ramírez, 2015).

Con base a todas estas fundamentaciones, el análisis de supervivencia con un conjunto de variables explicativas se desarrolla con el modelo de regresión de riesgos proporcionales de Cox, para determinar los factores de pronóstico de peligro de fallecer (Martínez-Baz et al., 2018). Es así que (Téllez et al., 2019), examinaron la relación entre el tipo de admisión hospitalaria, supervivencia y características patológicas y demográficas tales como: edad, genero, localización del tumor, estado patológico, quimioterapia previa cirugía y supervivencia del paciente que están asociadas significativamente al cáncer colorrectal. Las tasas de supervivencia se calcularon desde las fechas del diagnóstico hasta la fecha de recurrencia o muerte del paciente bajo el análisis de la Regresión de Cox aplicado para determinar la supervivencia global y supervivencia libre de enfermedad. Este modelo multivariante nos permite evaluar las correlaciones de las diferentes características clínicas de los pacientes con cáncer colorrectal, lo que coadyuva a pronosticar el riesgo que produce este cáncer. Además, efectuó comparaciones con los pacientes en los diferentes grados patológicos y los estadios TNM; llegando a establecer que la estadificación TNM es un factor pronóstico independiente (Du et al., 2017).

En base a lo expuesto la presente investigación abordará como objetivo efectuar el análisis de la supervivencia en pacientes con cáncer colorrectal mediante el Modelo de Regresión de Cox en la Provincia de Manabí, Ecuador, y que este modelo multivariante nos permita establecer la supervivencia a 6 años de los pacientes que recibieron su diagnóstico de este cáncer en el periodo enero del 2010 a diciembre del 2015 y su conexión significativa con las variables edad, sexo, estadio de la enfermedad, supervivencia y los meses de tratamiento, a su vez entregue resultados valiosos a la comunidad médica regional, nacional e internacional para que logren establecer estrategias para disminuir la incidencia, morbilidad y mortalidad de este cáncer, y así aumentar la 
condición de la calidad de vida de las personas afectadas $y$ en las determinaciones del galeno con el paciente.

\section{Metodología (Materiales y métodos)}

El presente estudio de investigación es descriptivo y explicativo de un diseño no experimental de tipo transversal-descriptivo, se analizó de manera retrospectiva información de una base de datos de 194 pacientes con cáncer colorrectal, que tuvieron confirmada su enfermedad en hospital oncológico Julio Villacreses Colmont de la provincia de Manabí. Para la obtención de la información, los datos de cada paciente fueron incluidos en la ficha técnica del departamento de estadística de Solca Manabí, que luego bajo criterios de inclusión y exclusión se obtuvo una data de 129 pacientes, posteriormente se diseña una matriz con las variables que serán las que intervengan en la investigación de la supervivencia en pacientes con cáncer colorrectal en la Provincia de Manabí, para lo cual se construye una tabla con el análisis descriptivo desarrollando una exploración lo más minuciosa factible del cáncer colorrectal, exceptuando obtener cuales son los motivos y efectos que estas causan. Valoramos de manera porcentual cada característica, se examinó la codificación y las transformaciones que constituyen esta dolencia, sin efecto de pararse a reflexionar o estimar acerca de ellas.

Para efecto de esta investigación, se registran variables que priman en los pacientes características tales como: edad, sexo, estadio de la enfermedad, supervivencia, meses de tratamiento que viene hacer la variable respuesta, cuyo efecto es dar un mayor beneficio en el análisis de supervivencia, es así que según (Guevara, 2018), expresa que en el análisis de supervivencia nos posibilita distinguir el efecto del impacto de una o más variables independientes 0 covariables, siempre y cuando la variable dependiente logra formularse en términos de tiempo hasta que ocurra el hecho final o evento, es decir es un examen de alerta temprana, proveniente de la verificación estadística de calidad, su análisis de supervivencia se lo efectuó con el 
programa RStudio versión 3.2 .2 (Team, 2018).

\section{Resultados}

En el desarrollo de la investigación se observaron 196 pacientes diagnóstico de cáncer de colorrectal, que bajo criterios de inclusión y por decisión técnica del investigador, permanecieron 129 pacientes para el análisis de supervivencia, se localizó una superioridad del sexo femenino con $55,81 \%$ y el masculino con $44,19 \%$, con un intervalo entre $14 \mathrm{y}$ 97 años, la media fue de 61,6 años. El grupo etario donde se agrupan la cantidad superior de pacientes es el de 60 a 78 años con $40,31 \%$ y el inferior de iguales o menores de 20 años con $1,55 \%$. El $49,61 \%$ poseen un TNM 3 y el 27,13\% con un TNM x. El $40,31 \%$ se han tratado durante menos de dos años este cáncer y el $17,37 \%$ más de cinco años. El $56,59 \%$ siguieron vivos y el $43,41 \%$ fenecieron (Tabla 1).

Posteriormente se efectuó el análisis de supervivencia general, analizando el tiempo de falla y de censura, situándose como la variable respuesta meses de tratamiento en esta investigación, cuantificada en meses desde inicio diagnóstico hasta el fin del estudio o suceso de interés ósea la muerte del paciente con cáncer colorrectal. El tiempo más alto de supervivencia de los 56 pacientes que alcanzaron el suceso, es de 20 meses para 16 pacientes (Figura 1).

En el análisis del tiempo de supervivencia en los pacientes objeto de estudio, se mostraron datos parciales, lo que nos indica censuras múltiples de tipo I a la derecha (tiempo censurado); esto implica que el comienzo del tiempo de supervivencia ocurrió en diferentes fechas y a la fecha de finalización del estudio no ocurrió el hecho de interés (muerte); la salida de estos pacientes no ocurre por motivos enlazados a la terapia o la variable de agrupación, porque ciertos pacientes murieron después del corte de finalización del estudio. En los intervalos de 0 a 10 y 30 a 40 meses existe el tiempo más alto donde los pacientes no llegaron al hecho de interés (muerte) (Figura 2).

En la (Tabla 2) las variables de la ecuación exponen los valores exponencial de Beta asemejados al hazard ratio que identifica la medida de riesgo, en este caso los 
significativos son valores negativos que son los estadios de la enfermedad (T2, T3) en base a los resultados son factores bajos de riesgo de este cáncer, lo que implica que los pacientes en estadio (T2) con un $95 \%$ de confianza (IC95\%:0.017-0,909) tienen 2,1 veces de mayor supervivencia en los 72 meses de seguimiento, en relación con el estadio de enfermedad $T x$, mientras que los pacientes con estadio (T3) (IC95\%:0,196-0,669) tienen 1,07 veces de mayor supervivencia en los 72 meses de seguimiento, en relación con el estadio de enfermedad Tx. Posteriormente el estadístico chi cuadrado reformado de Wald es significativo con valores $p>0,040$ y $p>0,001$ respectivamente para los estadios de la enfermedad (T2 y T3. En relación con las covariables edad y sexo entregaron valores no significativos para el riesgo en este cáncer, por lo tanto no inciden en el desenlace del hecho de interés; es decir llegar a estadios tempranos es de protección y evaluar el tumor principal a tiempo en este cáncer te protege contra la muerte.
La curva de supervivencia global (Figura 3) nos indica el camino que desarrollan los 129 pacientes con cáncer colorrectal en llegar al hecho de interés durante un lapso de tiempo determinado. La posibilidad de una supervivencia global a los 6 años fue de $15 \%$, esto nos indica que para 72 meses de seguimiento aproximadamente el $15 \%$ pacientes continúan vivos, claramente establece que más del $85 \%$ de los pacientes estudiados murieron a los 72 meses que aborda el estudio.

Las curvas de supervivencia ajustadas por la edad y el sexo en relación con cada uno de los estadios de la enfermedad nos indican como va disminuyendo la supervivencia en los pacientes con cáncer colorrectal en relación con TNM (T2, T3, T4 y Tx). Es evidente observar que los pacientes que llegan a estadios (Tx y T4) tienen menor tiempo de vida en relación con los estadios clasificados como menos avanzados En el mes 72 aproximadamente con el $2 \%$ y $4 \%$ de los pacientes en el estadio Tx y T4 estarían vivos, mientras que cerca del $55 \%$ de los pacientes en estadio T1 estarían todavía con vida (Figura 4). 
El grafico de la (Figura 5) indica que aumentan con mayor cantidad en las funciones de riesgo entre grupos relación con los periodos de tiempo, son proporcionales al tiempo, donde por ende es donde a mayor estadio observamos que las curvas para los de la enfermedad, el riesgo de estadios T2, T3, T4 y Tx, donde refleja que los estadios Tx y T4 supervivencia al cáncer colorrectal será también mayor.

\section{Figuras y Tablas}

Figura 1. Tiempo de supervivencia.

\section{T.Superv.}

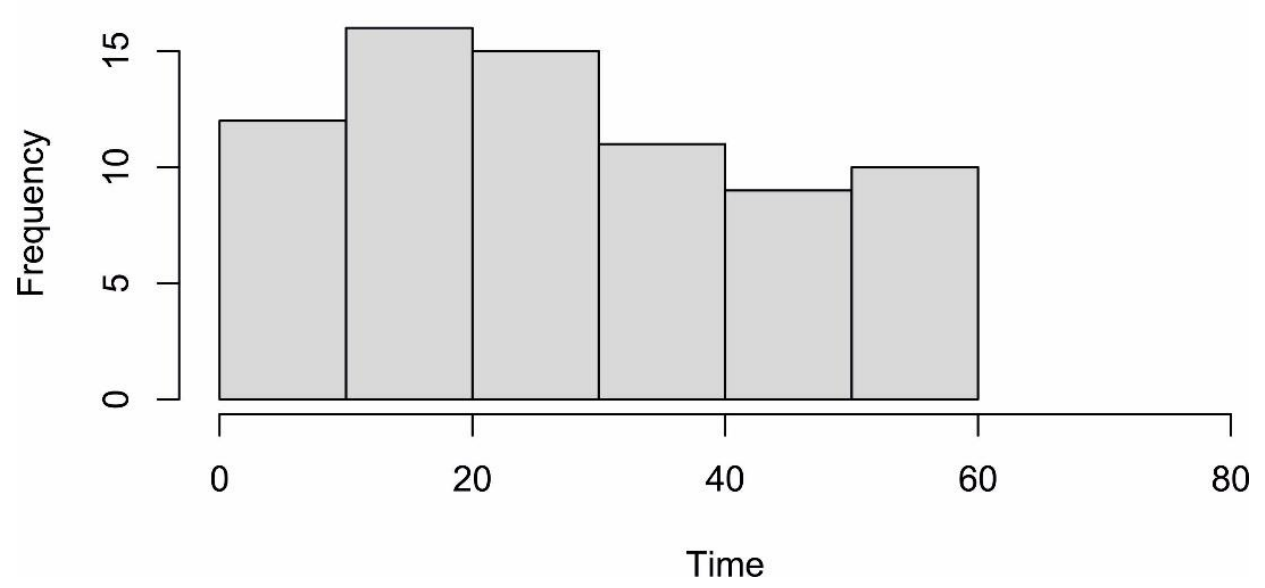

Figura 2. Tiempo censurado.

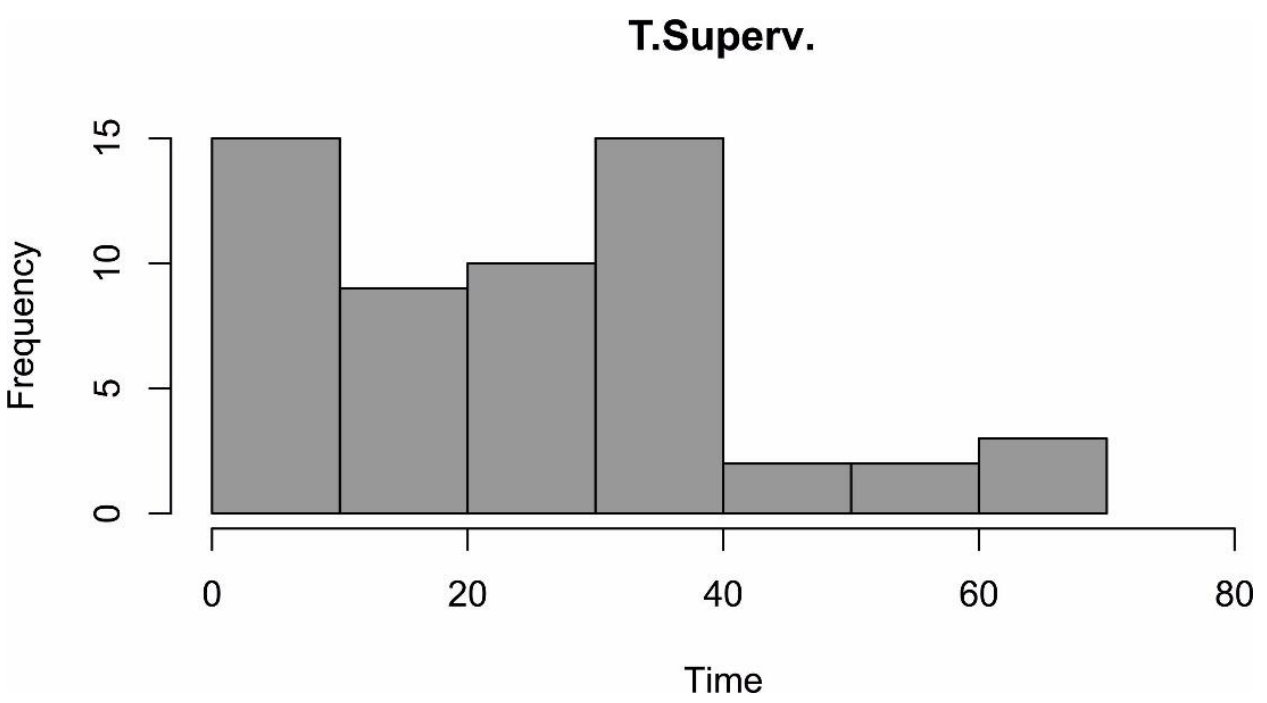


Figura 3. Curva de supervivencia ajustada por los cofactores.

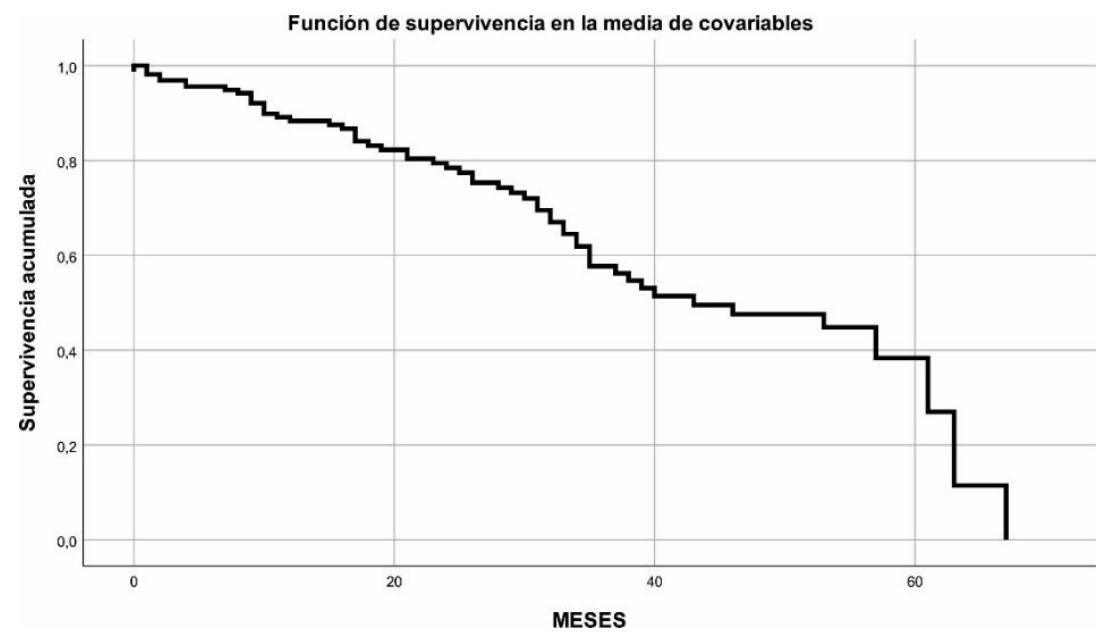

Figura 4. Curva de supervivencia por estadios de la enfermedad.

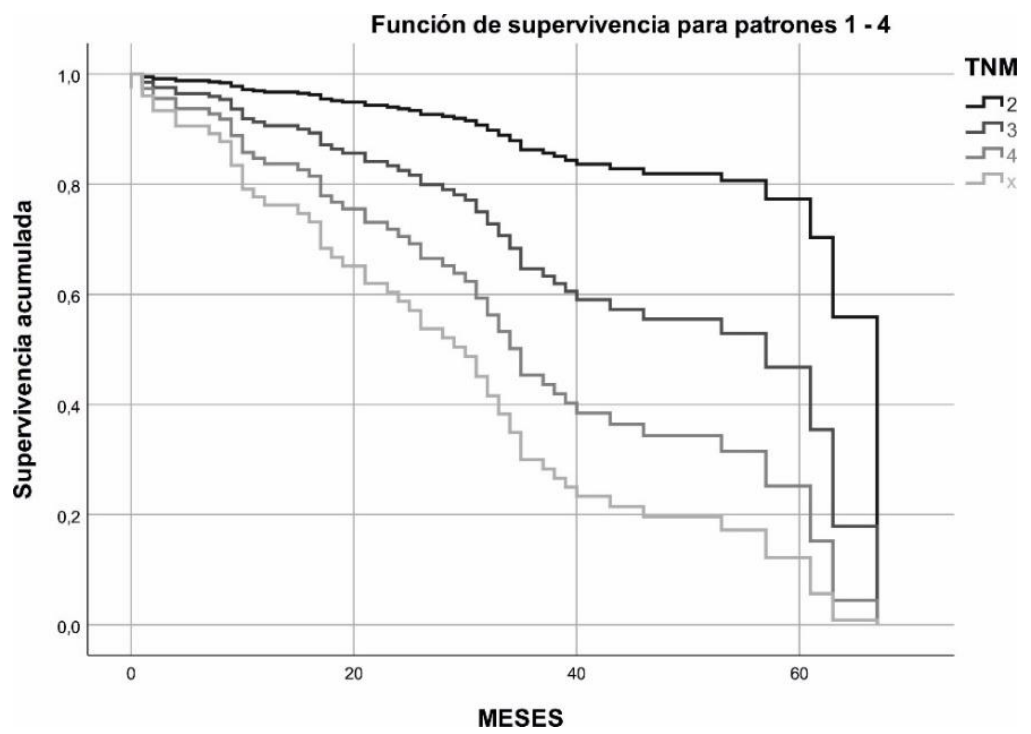

Figura 5. Evaluación de la función de riesgos proporcionales.

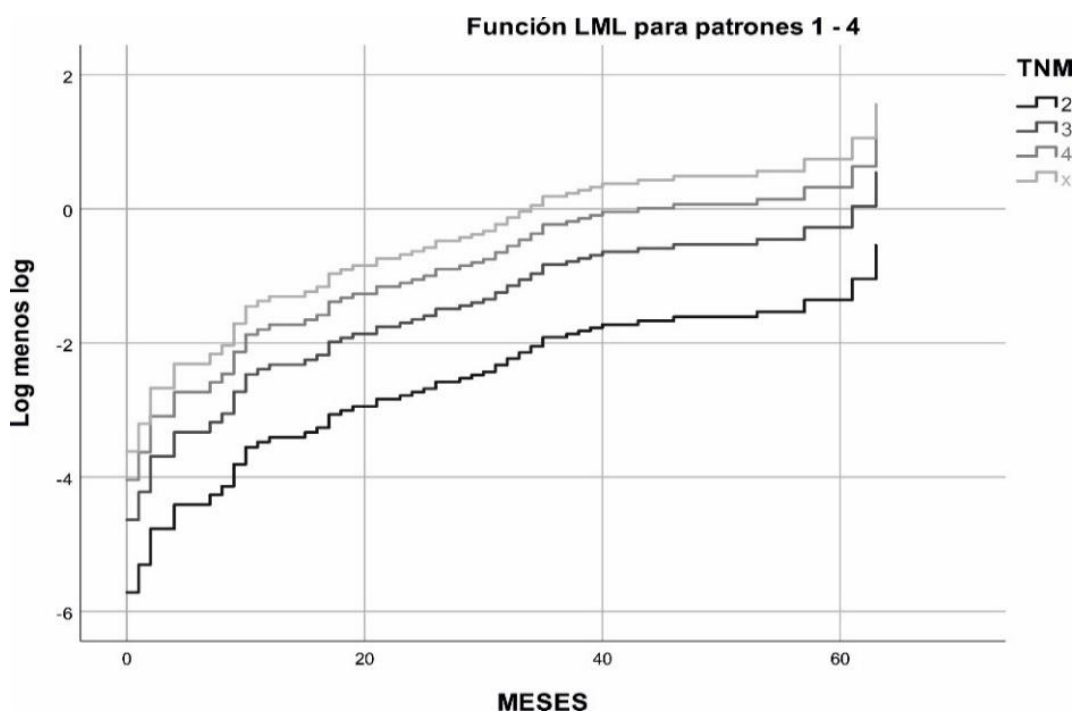


Tabla 1. Descripción de variables

\begin{tabular}{|c|c|c|c|}
\hline \multicolumn{4}{|c|}{ DESCRIPCION DE VARIABLES } \\
\hline VARIABLE & DESCRIPCIÓN & CODIFICACIÓN & $\%$ \\
\hline \multirow{2}{*}{ Género } & \multirow{2}{*}{ Género } & Masculino (1) & 44,19 \\
\hline & & Femenino (2) & 55,81 \\
\hline \multirow{5}{*}{ Edad } & \multirow{5}{*}{ Edad en años } & $<=20$ años & 1,55 \\
\hline & & 21-39 años & 10,08 \\
\hline & & 40-59 años & 31,01 \\
\hline & & 60-78 años & 40,31 \\
\hline & & 79- más años & 17,05 \\
\hline \multirow{2}{*}{ Sobrevida } & \multirow{2}{*}{ Sobrevida } & Vivo (1) & 56,59 \\
\hline & & Muerto $(0)$ & 43,41 \\
\hline \multirow{4}{*}{ Estadio del Tumor } & \multirow{4}{*}{ TNM } & Tamaño 2 (2) & 10,55 \\
\hline & & Tamaño 3 (3) & 49,61 \\
\hline & & Tamaño 4 (4) & 12,40 \\
\hline & & Tamaño x (x) & 27,13 \\
\hline \multirow{4}{*}{ Meses } & \multirow{4}{*}{ Meses de Tratamiento } & $<=20$ & 40,31 \\
\hline & & $21-37$ & 21,84 \\
\hline & & $38-55$ & 18,86 \\
\hline & & 56- más meses & 17,37 \\
\hline
\end{tabular}

Tabla 2. Factores predictores de supervivencia de los pacientes con cáncer colorrectal.

\begin{tabular}{|c|c|c|c|c|c|c|c|c|}
\hline & \multirow{2}{*}{ B } & \multirow{2}{*}{ SE } & \multirow{2}{*}{ Wald } & \multirow{2}{*}{ df } & \multirow{2}{*}{ Sig. } & \multirow{2}{*}{$\operatorname{Exp(B)}$} & \multicolumn{2}{|c|}{$\mathbf{9 5 , 0 \%}$ CI para $\operatorname{Exp(B)}$} \\
\cline { 7 - 9 } & & & & & & & Inferior & Superior \\
\hline SEXO &, 409 &, 276 & 2,196 & 1 &, 138 & 1,506 &, 876 & 2,587 \\
\hline TNM & & & 13,212 & 3 &, 004 & & & \\
\hline TNM (1) & $-2,100$ & 1,023 & 4,218 & 1 &, 040 &, 122 &, 017 &, 909 \\
\hline TNM (2) & $-1,017$ &, 314 & 10,487 & 1 &, 001 &, 362 &, 196 &, 669 \\
\hline TNM (3) &,- 421 &, 372 & 1,282 & 1 &, 257 &, 656 &, 316 & 1,361 \\
\hline EDAD &,- 017 &, 009 & 3,788 & 1 &, 052 &, 983 &, 966 & 1,000 \\
\hline
\end{tabular}

\section{Conclusiones}

En el campo del examen descriptivo explicado en el presente estudio sobre el cáncer colorrectal, sobre características tales como: sexo, edad, estadio del tumor, supervivencia, meses de tratamiento; logran evidenciar el proceder de este conjunto de variables en el desarrollo de la investigación y ayudaron abrir el camino a una investigación amplia y sostenible sobre la supervivencia del cáncer colorrectal en la Provincia de Manabí. 
En lo que respecta al análisis de la supervivencia general proporciono conocimiento estadístico relevante de los datos censurados; es decir los pacientes que no llegaron al hecho o suceso (73 pacientes); de la misma forma las fallas identificaron el lapso de tiempo de la supervivencia de los 56 pacientes lograron llegar al hecho o suceso.

La aplicación del modelo de riesgos proporcionales de Cox aporto con la significancia estadística del modelo de la investigación, así mismo presento las salidas de las curvas de supervivencias enfocada en las entradas de las covariables cuantitativas y cualitativas objeto del estudio. Donde las covariables estadio del tumor en su estadificación T2 y T3 representaron factores de protección en relación al estadio del tumor en su estadificación $T x$, ya que aproximadamente el $57 \%$ y $19 \%$ de los pacientes respectivamente están vivos al finalizar los 72 meses; en cambio en relación al estadio de la enfermedad en su estadificación T4 aproximadamente un $4 \%$ continúan vivos; esto quiere decir que los estadios de la enfermedad (tamaño del tumor) constituyen factores en un rango de predicción en la supervivencia al cáncer colorrectal en Manabí.

De aquí la importancia de sumar esfuerzos y establecer estrategias que ayuden a una detención más temprana, como guías, charlas sobre como efectuar esta detención a tiempo, ya que su efecto será benéfico sobre la mortalidad en cáncer colorrectal en Manabí, lograr cesar este cáncer en estadios más tempranos y que se pueda evaluar el tumor principal nos llevaría a una baja recurrencia y a su vez disminuir la mortalidad.

Este tipo de modelos como el Cox son beneficiosos en el área de la salud, en zonas vinculadas con enfermedades graves y de alta mortalidad, su facultad de predicción permite emplear distintas medidas de muestras en supervisar y procesar datos de supervivencia.

\section{Bibliografía}

Castillo Ramírez, A. V. (2015). Cáncer Colorrectal. Universidad Técnica de Ambato-Facultad de Ciencias de la Salud-Carrera de ....

Corral, F., Cueva, P., Yépez. José, \& Tarupi,

W. (2018). 
Tendencias en incidencia y mortalidad por cáncer durante tres décadas en Quito Ecuador. Colombia Médica, 7. https://doi.org/10.25100/cm.v $49 i 1.3785$

Du, P., Xu, B., Zhang, D., Shao, Y., Zheng, X., Li, X., Xiong, Y., Wu, C., \& Jiang, J. (2017). Hierarchical investigating the predictive value of p53, COX2,EGFR, nm23 in the post-operative patients with colorectal carcinoma. Oncotarget, 8(1), 954-966. https://doi.org/10.18632/oncot arget.13512

Guevara, L. (2018). El Análisis de Supervivencia en la Gestión del Riesgo de Crédito: El Caso de una Cartera Hipotecaria Ecuatoriana.

Martínez-Baz, I., Delfrade, I., \& Etxeberria, J. (2018). Characteristics and statistical methods used in original articles published in public health journals in Spain [Características y métodos estadísticos empleados en los artículos originales publicados en las revistas de salud pública en España]. Anales Del Sistema Sanitario de Navarra, 41(3), 8. https://doi.org/doi.org/10.239 38/ASSN.0382

Ministerio de Salud Pública. (2017). Estrategia Nacional para la atención integral del cáncer en el Ecuador.
Oviedo, D. (2015). Supervivencia de cáncer de colon en una institución Oncológica privada de la ciudad de manizales,2003-2010.

Team, R. (2018). R Studio - Google Académico.

RStudio: Integrated Development for R. RStudio, Inc. Boston, MA.

Téllez, T., García, M., Zarcos, I., Rivas, F., Pérez, E., Padilla, M., Baré, M., Morales, M., Rueda, A., Alcaide, J., \& Redondo, M. (2019). First hospital contact via the Emergency Department is an independent predictor of overall survival and diseasefree survival in patients with colorectal cancer. Revista española de Enfermedades Digestivas : Órgano Oficial de La Sociedad española de patología Digestiva, 111(10), 750-756.

https://doi.org/10.17235/reed. 2019.5777/2018

Villanueva-Pájaro, D. J., VergaraDagobeth, E. E., SuárezCausado, A., \& Gómez-Arias, R. D. (2020). Epidemiología de la interrelación cáncer colorrectal y diabetes mellitus tipo 2. Revisión sistemática. Revista Facultad Nacional de Salud Pública, 38(2), 21. https://doi.org/10.17533/udea .rfnsp.e337048 